\title{
Morphology Control in 2D Carbon Nitrides: Impact of Particle Size on Optoelectronic Properties and Photocatalysis
}

\author{
Julia Kröger, Alberto Jiménez-Solano, Gökcen Savasci, Vincent W. h. Lau, Viola Duppel, Igor \\ Moudrakovski, Kathrin Küster, Tanja Scholz, Andreas Gouder, Marie-Luise Schreiber, Filip Podjaski, \\ Christian Ochsenfeld, Bettina V. Lotsch*
}

\begin{abstract}
J. Kröger, Dr. A. Jiménez-Solano, G. Savasci, V. Duppel, Dr. I. Moudrakovski, Dr. K. Küster, Dr. T. Scholz, A. Gouder, M.-L. Schreiber, Dr. F. Podjaski, Prof. C. Ochsenfeld, Prof. B. V. Lotsch Max Planck Institute for Solid State Research, Heisenbergstr. 1, 70569 Stuttgart, Germany E-mail: b.lotsch@fkf.mpg.de

J. Kröger, G. Savasci, A. Gouder, Prof. C. Ochsenfeld, Prof. B. V. Lotsch Department of Chemistry, University of Munich, LMU, Butenandtstr. 5-13, 81377 Munich, Germany

J. Kröger, G. Savasci, Dr. F. Podjaski, Prof. C. Ochsenfeld, Prof. B. V. Lotsch

Cluster of excellence e-conversion, Lichtenbergstr. 4a, 85748 Garching, Germany

Dr. V. W. h. Lau

Department of Materials Science and Engineering, Korea University, Seoul 02841, Republic of Korea
\end{abstract}

Key-words: carbon nitrides, poly(heptazine imide), photocatalysis, trap states, defect tuning, particle size

\begin{abstract}
The carbon nitride poly(heptazine imide), PHI, has recently emerged as a powerful 2D carbon nitride photocatalyst with intriguing charge storing ability. Yet, insights into how morphology, particle size and defects influence its photophysical properties are virtually absent. Here, ultrasonication is used to systematically tune the particle size as well as concentration of surface functional groups and study their impact. Enhanced photocatalytic activity correlates with an optimal amount of those defects that create shallow trap states in the optical band gap, promoting charge percolation, as evidenced by time-resolved photoluminescence spectroscopy, charge transport studies, and quantum-chemical calculations. Excessive amounts of terminal defects can act as recombination centers and hence, decrease the photocatalytic activity for hydrogen evolution. Re-agglomeration of small particles can, however, partially restore the photocatalytic activity. The type and amount of trap states at the surface can also influence the deposition of the co-catalyst $\mathrm{Pt}$, which is used in hydrogen evolution experiments. Optimized conditions entail improved Pt distribution, as well as an enhanced wettability and colloidal stability. A description
\end{abstract}


of the interplay between these effects is provided to obtain a holistic picture of the size-propertyactivity relationship in nanoparticulate PHI-type carbon nitrides that can likely be generalized to related photocatalytic systems.

\section{Introduction}

Graphitic carbon nitrides $\left(\mathrm{g}-\mathrm{C}_{3} \mathrm{~N}_{4}\right)$, of which the archetype material is known since the $19^{\text {th }}$ century as the heptazine-based polymer "melon", have recently re-entered the stage as an earth-abundant semiconductor system with potential application ranging from photo/electrochemical redox catalysis for environmental remediation to chemical sensing. ${ }^{[1]}$ These applications are based on the material's favorable properties, including chemical and thermal stability, an optical band gap in the visible range and energy levels appropriately positioned for a number of key photocatalytic reactions, including water splitting. ${ }^{[2]}$ The class of carbon nitrides comprises triazine- and heptazine-based polymers, the dimensionality of which being determined by the synthesis procedure: whereas 1D heptazine-based polymers are obtained by heating the precursors in a solid state reaction, ionothermal synthesis results in the 2D network poly(heptazine imide) (PHI) or poly(triazine imide) (PTI) ${ }^{[3-5]}$ For example, using a KSCN melt as a reaction medium, the PHI structure is obtained with hydrated potassium ions incorporated from the melt (K-PHI) and possessing cyanamide groups as defects; the potassium ions can be subsequently exchanged e.g. by protons, forming H-PHI. ${ }^{[3,6,7]}$ Besides high photocatalytic hydrogen evolution rates, ${ }^{[6,7]} \mathrm{PHI}$ exhibits the unusual ability to stabilize photo-generated electrons after the holes are quenched by suitable electron donors. This can be exploited afterward to temporally decouple for hours the absorption of sunlight from its transformation into chemical fuels such as hydrogen. ${ }^{[3,8]}$ This longlived photo-reduced state can also be harvested for time-delayed electrical discharge after illumination in an aqueous solar battery anode. ${ }^{[9]}$ Unveiling the full potential of such "lightstorage" properties necessitates a more thorough understanding of the structure-property-activity relationships in PHI. Even though the atomic-level structure of K- and H-PHI has recently been resolved, ${ }^{[3]}$ little is known about the impact of morphology, particle size, and surface terminations on the photocatalytic properties. In particular, surface terminations were recently described to have a significant impact on the photocatalytic behavior of PHI by enhancing its interaction with reactants. ${ }^{[10]}$ 
For melon-type carbon nitrides, it was reported that morphology tuning, e.g. by exfoliation, can change the optical and electronic properties of the material, which then results in enhanced photocatalytic activity. ${ }^{[11,12,13]}$ Different reasons for this enhancement were listed, including increased surface area, higher amount of beneficial defects or surface functional groups for attaching the co-catalyst as well as higher porosity, leading to enhanced light absorption and charge transport, which results in a reduction of the recombination rate of photo-generated charges. ${ }^{[13-15]}$ This demonstrates the importance of morphology and surface properties for photocatalytic reactions and the need to understand their correlations. However, there are not only reports about enhanced photocatalytic behavior by reducing the particle size, but also about reduced charge carrier lifetimes in exfoliated carbon nitride, which then lead to a reduced hydrogen evolution rate. ${ }^{[12,16]}$ Consistent with these findings, a more efficient charge carrier separation with longer lifetimes of photoinduced charges was reported for carbon nitrides, since the excitons were found to dissociate across the layers rather than within one layer, posing the question of whether excitons in carbon nitrides are inter- or intralayer excitons, or both. ${ }^{[12,17,18]}$ The above highlights the controversial nature and a lack of understanding of the size-activity relationship in carbon nitrides, which is influenced by many parameters. One possible explanation for these conflicting results may be found in the differences in synthesis and exfoliation of melon, the latter carried out e.g. by sonication, thermal treatment, mechanical treatment, ion intercalation, or chemical exfoliation by sulfuric acid. ${ }^{[14,19]}$ During the exfoliation process, defects or vacancies can be generated, and new surface terminal groups may develop on the carbon nitride backbone. On the one hand, defects are discussed as facilitators in photocatalysis. They can form electronic states in the optical band gap, which can enhance visible light absorption and influence charge carrier transfer at the interface. ${ }^{[20,}$ ${ }^{21,22]}$ On the other hand, defects can act as recombination centers or deep trap states, reducing the charge carriers' mobility and driving force for photocatalytic reactions, which counteracts photocatalytic performance. ${ }^{[2,23-25]}$ A recently published work highlights the effect of functional groups and defects in melon-type carbon nitride being beneficial only up to a particular concentration, which also strongly depends on the type of functional group or defect. ${ }^{[26]}$ This ambiguity of structure and defect types warrants an in-depth analysis of the interplay and influence of morphology and structural changes on different optoelectronic properties and, consequently, photocatalytic activity. As photocatalysis is a complex process relying on intertwined structural, optoelectronic, and morphological properties, many of which are associated with different time 
scales, ${ }^{[27]}$ disentangling these factors is essential for understanding the overall photocatalytic activity.

Here, we study the effect of ultrasonic particle size reduction of $\mathrm{K}$ - and $\mathrm{H}-\mathrm{PHI}$ on the photocatalytic hydrogen evolution reaction (HER) by analyzing multiple parameters. Central to our study is the size separation of PHI particles, based on differential centrifugation velocities. By breaking apart agglomerates, functional surface groups and defects are generated and, comprehensively analyzed by various techniques. With decreasing particle size and the correspondingly increase in surface area, the number of functional groups increases (especially $\mathrm{NH}_{2}, \mathrm{OH}$, and $-\mathrm{C}=\mathrm{O}$ ), while at the same time, light absorption is slightly shifted to longer wavelengths. Besides, we find that with increasing amounts of functional groups, the distribution of photo-deposited Pt, used as co-catalyst for HER, becomes more uniformly distributed, and the Pt particle size is reduced. Simultaneously, the suspension stability is enhanced, which is beneficial for high photocatalytic activity, provided that the particles are not smaller than $180 \mathrm{~nm}$. On the other hand, the amount and depth of trap states increases with the number of functional groups, influencing the accessibility of photo-excited electrons for transfer to the surface. Intriguingly, we observe that re-agglomeration of those particles causes a significant increase of the hydrogen evolution rate (up to $35 \%$ ). This might be correlated with an enhanced crystallite interaction in the particle when the amount of functional groups increases, enabling better charge carrier separation. Photoluminescence (PL) and conductivity measurements hint to a multiple trapping and release model (MTR) ${ }^{[28]}$ for the electron transfer in PHI. This observation points to opposing effects of reduced charge carrier separation and enhanced dispersion stability with decreasing particle size. Therefore, hydrogen evolution as a function of particle size is found to be an interplay of all these parameters. Optimal performance is found for $2 \mathrm{~h}$ sonicated samples with particle sizes above $180 \mathrm{~nm}$, ideally in a re-agglomerated material. Importantly, we find that the contribution of each parameter, depending on the particle size, is not as unambiguous as often reported in the literature.

\section{Results and Discussion}

\section{Morphology and structural analysis}

Particle size reduction by an ultrasonication route involved the sonication of PHI in water for an extended duration of $2 \mathrm{~h}$, followed by fractionated centrifugation of the aggregates. This yields a colloidal solution with a concentration of around $5 \mathrm{mg} \mathrm{mL}^{-1}$ (Figure 1a), akin to procedures 
reported in literature.$^{[4,9]}$ The first precipitate (P1) contains the largest particles (P1: 300-1500 nm), the second precipitate $(\mathrm{P} 2)$ contains medium particle sizes $(\mathrm{P} 2: 100-400 \mathrm{~nm})$, with the third precipitate (P3) containing the smallest particles down to $40 \mathrm{~nm}$ (Figure S1-S3, Table S4, Supporting Information). The particle sizes were analyzed by a combination of dynamic light scattering (DLS), atomic force microscopy (AFM), transmission electron microscopy (TEM), and scanning electron microscopy (SEM), as well as Brunauer-Emmett-Teller (BET) surface area analysis (Figure S1-S10, Supporting Information). To reduce the particle sizes even further, HPHI was sonicated for $24 \mathrm{~h}$, and the supernatant (P4) was separated and precipitated at 500 relative centrifugal force (RCF) (Table S1, Supporting Information). The resulting particle sizes are in the range of 20-350 nm (Figure S3, S6, Supporting Information). Since K-PHI is protonated when storing in water for a long time, forming $\mathrm{H}-\mathrm{PHI}$, this $24 \mathrm{~h}$ sonication process was only performed on H-PHI (Figure S44, Supporting Information). The suspension of PHI with small particles (below $200 \mathrm{~nm}$ ) remained colloidally stable in water for months, with no indication of settling. This is also evident by their large zeta-potential ( $-23 \mathrm{mV}$ to $-45 \mathrm{mV}$ ) across all $\mathrm{pH}$ values measured for H-PHI sheets (Figure S9, Table S2 Supporting Information). The colloidal suspensions can be dried to isolate PHI for further characterization (notated as "PHI re-agglomerate").

The primary crystallite size obtained by TEM analysis of pristine particles is only in the range of 20-45 nm (Figure S4, Supporting Information) when compared to SEM and AFM analysis, which show that larger particles are agglomerates of smaller crystallites. After $24 \mathrm{~h}$ sonication (P4), the primary crystallite size is reduced to $5-25 \mathrm{~nm}$, as shown by TEM (Figure 1b, Figure S5, Supporting Information). In AFM, a minimal thickness of 3-5 nm (Figure 1c, d) for P4 particles could be measured. Based on the interlayer separation of $3.16 \AA$ resulting from the PXRD analysis (vide infra), this corresponds to 9 to $16 \mathrm{PHI}$ layers in the stacking direction. Crystallite sizes can also be estimated from PXRD data (Figure 2a) by using the Scherrer equation (Table S4, Supporting Information), which gives a more representative value of the average domain size compared to the local information gained from TEM or AFM. For particles being sonicated for $24 \mathrm{~h}$, the crystallite size is only slightly reduced (by $15 \%$ to $22( \pm 1) \mathrm{nm}$ in the vertical direction) with respect to the pristine material (lateral: $14( \pm 1) \mathrm{nm}$ and vertical: $26( \pm 1) \mathrm{nm}$ ), proving that primary crystallites remain intact during sonication, but that the agglomerates break apart, forming smaller agglomerates or even single crystallite particles. 
The measurement of the hydrodynamic diameter of the particles, estimated by DLS (Figure S10, Table S3, Supporting Information), is in reasonable agreement with particle sizes of K-PHI and H-PHI obtained by microscopy techniques (Table S4, Supporting Information). However, since DLS analysis assumes perfectly spherical particles, a direct relationship between the results of microscopy and DLS for 2D materials is yet to be accurately determined. ${ }^{[29]}$ Nevertheless, DLS measurements were used in the following for particle size estimation, since this measurement allows for fast and quantitative particle analysis.

The analysis of BET surface area and pore volume reveals a type IV isotherm, associated with condensation processes in mesopores, most probably located between agglomerated particles as textural pores (Figure S11, Supporting Information). ${ }^{[30]}$ The surface area is slightly increased for H-PHI P4 $\left(70 \mathrm{~cm}^{3} \mathrm{~g}^{-1}\right)$ in comparison to the pristine material H-PHI $\left(60 \mathrm{~cm}^{3} \mathrm{~g}^{-1}\right)$ (Table S5, Supporting Information). This finding is in line with the assumption of particles consisting of agglomerated crystallites, where the big agglomerated particles break apart during sonication, forming smaller agglomerates or even single crystallites.
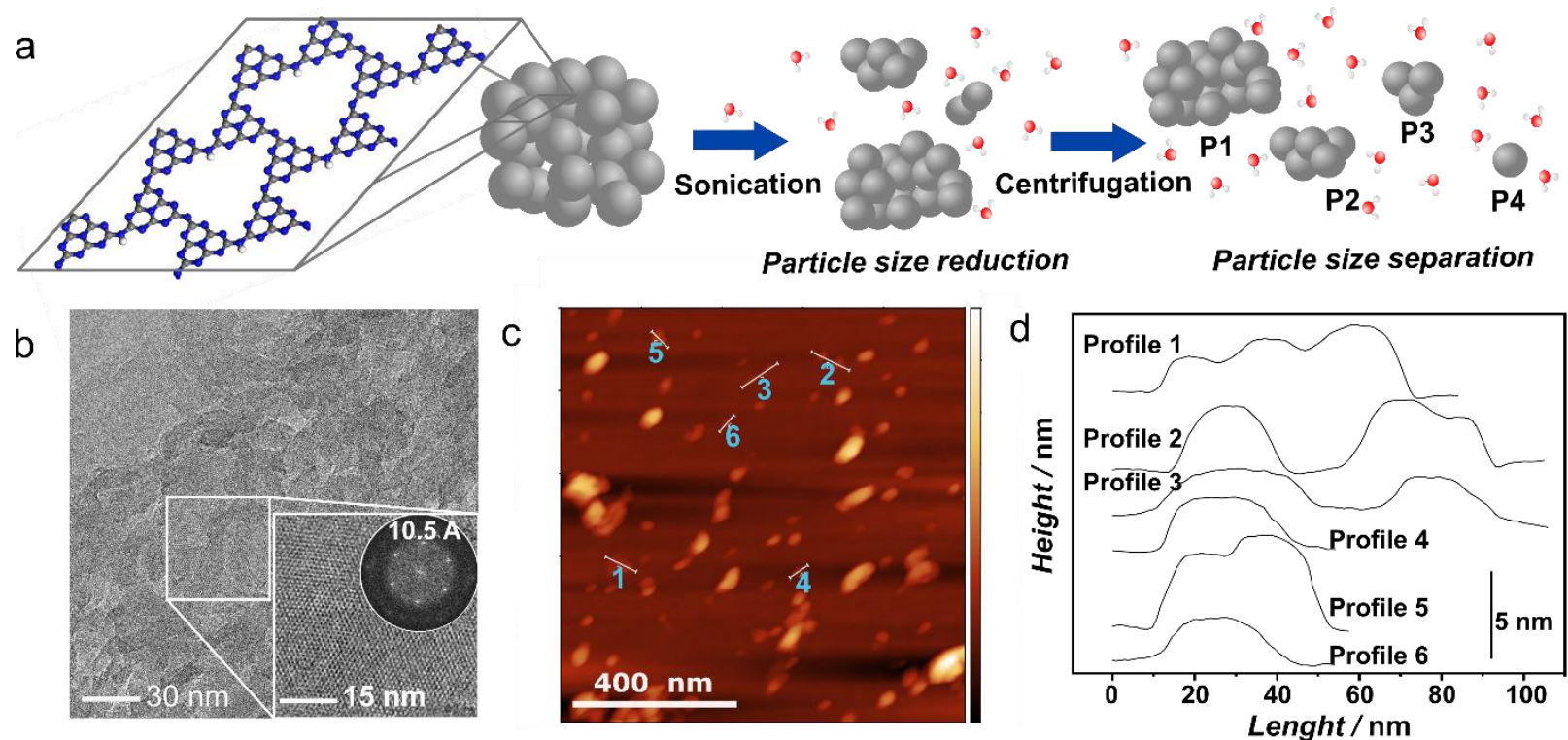

Figure 1: Morphology and particle size analysis of PHI. a) Schematic illustration of PHI sonication for breaking apart agglomerated particles, followed by stepwise centrifugation for particle size separation (blue: nitrogen, grey: carbon, white: hydrogen, red: oxygen). b) TEM image of H-PHI P4 ( $24 \mathrm{~h}$ sonicated), the inset showing a magnification of single P4 particles with the Fast Fourier Transformed (FFT) image showing the characteristic distances in PHI. c) AFM image of single H-PHI P4 particles (24 h sonicated). d) Corresponding height profiles.

Although considered a mild dispersion technique, acoustic bubbles formed by ultrasound are hot and can generate highly reactive radicals. ${ }^{[31]}$ Thus, the absence of chemical changes to the investigated materials must be ascertained. To verify that the sonicated materials are chemically 
identical to the pristine PHI and to study the surface termination of small particles, the colloidal solution was evaporated to dryness. The residual powder was structurally characterized by ${ }^{13} \mathrm{C}$ and ${ }^{15} \mathrm{~N}$ magic angle spinning solid state nuclear magnetic resonance spectroscopy (MAS NMR), Fourier transform infrared spectroscopy (FT-IR), powder X-ray diffraction (PXRD), elemental analysis, and X-ray photoelectron spectroscopy (XPS).

As stated above, the primary crystallite size is almost unchanged when the particle size is reduced by sonication. Therefore, in PXRD no significant changes are observed. The in-plane (110) and out-of-plane (001) reflections are at $7.9^{\circ}\left(11.10 \AA\right.$ ) and $27.9^{\circ}$ to $28.3^{\circ} 2 \theta$ (3.19 ̊ for H-PHI and $3.16 \AA$ for K-PHI), respectively, with the key difference being the slight isotropic broadening of the interlayer stacking reflection (Figure 2a, S12, S13, Supporting Information) in smaller particles. ${ }^{[3]}$

The FT-IR spectra of the colloidal H-PHI and K-PHI are basically identical to the pristine sample, showing all the signals corresponding to the heptazine moiety (Figure S14, S15, Supporting Information). This points clearly to an intact PHI polymer network, which is further proven by elemental analysis (Table S8, Supporting Information) as well as ${ }^{13} \mathrm{C}$ and ${ }^{15} \mathrm{~N}\{\mathrm{H}\} \mathrm{CP}$ MAS NMR spectroscopy (Figure $2 \mathrm{~b}, \mathrm{c}$ ). The signal positions in ${ }^{13} \mathrm{C}$ NMR at $157 \mathrm{ppm}$ to $158 \mathrm{ppm}$ (heptazinering carbon) and 163 to $164 \mathrm{ppm}$ (heptazine-ring carbon next to imide bridge) remain at the same position, but broadening of ${ }^{13} \mathrm{C}$ signals can be seen in all sonicated materials, likely due to surface functionalization or increased water interaction. The same trend can be observed in ${ }^{15} \mathrm{~N}\left\{{ }^{1} \mathrm{H}\right\} \mathrm{CP}$ NMR for the two main signals around -176 to $-190 \mathrm{ppm}$ (heptazine-ring nitrogen atoms) and -237 to $-244 \mathrm{ppm}$ (imide bridges). The peaks at $166 \mathrm{ppm}$ in the ${ }^{13} \mathrm{C}$ NMR and $-267 \mathrm{ppm}$ in the ${ }^{15} \mathrm{~N}$ NMR of K-PHI P1 are more pronounced than those in the pristine material, which also hints to an increase in $\mathrm{NH}_{2}$ groups, consistent with the literature ${ }^{[21]}$ (Figure 2b, c S24, Supporting Information). Additionally, an enhanced water interaction is visible in ${ }^{15} \mathrm{~N}$ NMR, especially in smaller particles, which is observed by a shift of the NH-bridge nitrogen peak at -240 ppm to -238 $\mathrm{ppm},{ }^{[3,4,32]}$ in agreement with quantum-chemical calculations (Figure S20-S23, Supporting Information). In line with this, the FT-IR analysis shows an increase in the O-H/N-H bond vibration at $3680-2220 \mathrm{~cm}^{-1}$ in atmospheric conditions (up to $47 \%$ in P4) with decreasing particle size, being more pronounced when the average particle size gets smaller than $200 \mathrm{~nm}$ (Figure 2d, S14-S18, Table S6-S7, Supporting Information). 

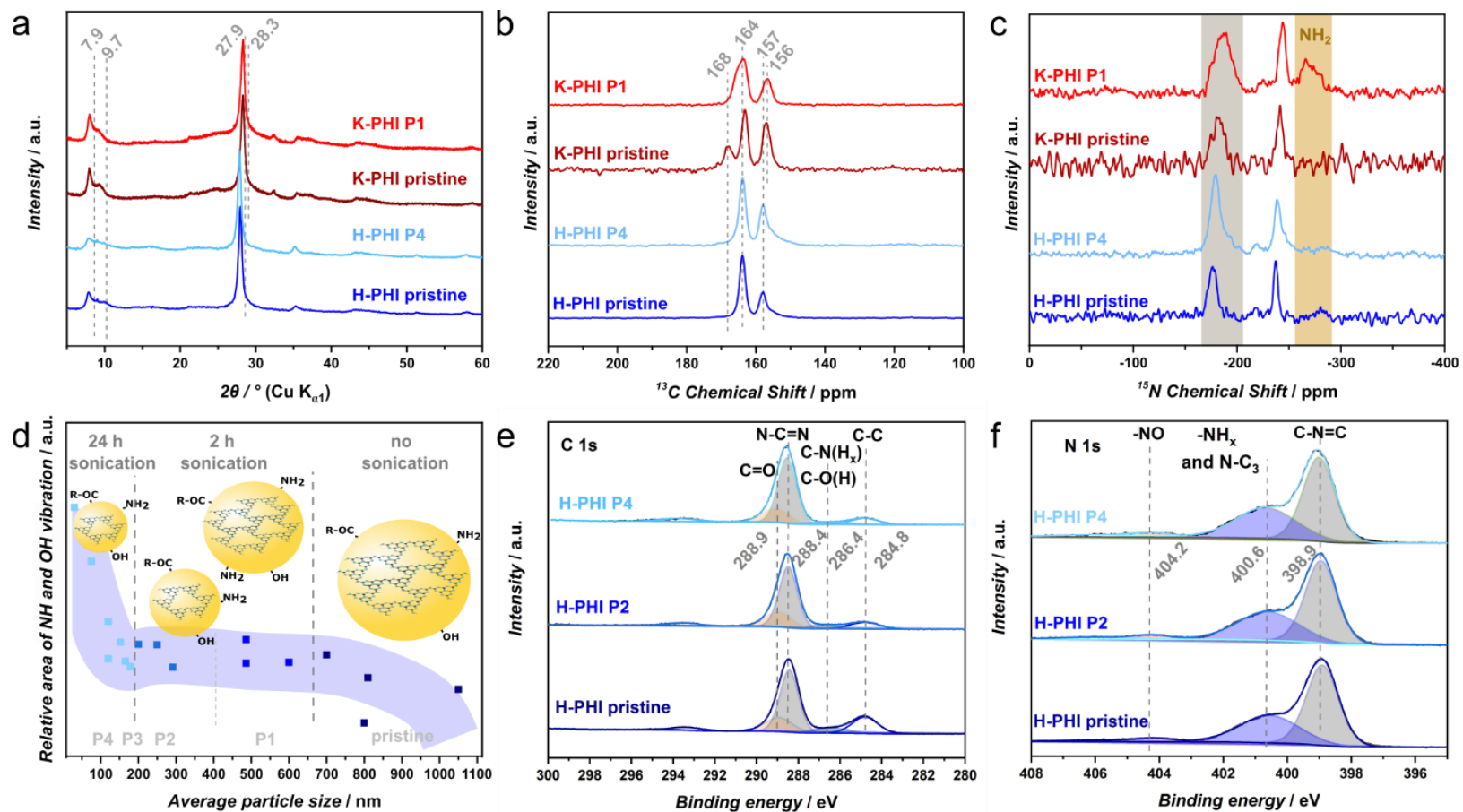

Figure 2: Characterization of structural defects of pristine PHI and small particles of PHI after sonication. a) PXRD of pristine KPHI and K-PHI P1 as well as pristine H-PHI and H-PHI P4. b) ${ }^{13} \mathrm{C}\left\{{ }^{1} \mathrm{H}\right\}$ CP MAS NMR experiments of pristine K-PHI and K-PHI $\mathrm{P} 1$ as well as pristine H-PHI and H-PHI P4. c) ${ }^{15} \mathrm{~N}\left\{{ }^{1} \mathrm{H}\right\}$ CP MAS NMR experiments of pristine K-PHI and K-PHI P1 as well as pristine H-PHI and H-PHI P4; d) Relative area of NH and $\mathrm{OH}$ vibrations (related to the heptazine vibration at $805-811 \mathrm{~cm}^{-1}$ ) of FTIR of $\mathrm{H}-\mathrm{PHI}$ as a function of particle size. $\mathrm{R}$ of carboxyl functionality $=\mathrm{OH}$ or $\mathrm{NH}_{2}$. e), f) XPS analysis of pristine $\mathrm{H}-\mathrm{PHI}$ (average particle size 1500-1000 nm) and H-PHI particles after $2 \mathrm{~h}(\mathrm{P} 2$ with average particle size 400-350 $\mathrm{nm}$ ) and $24 \mathrm{~h}$ sonication (P4 with average particle size around $79 \mathrm{~nm}$ ) e) C 1s spectrum, and f) N 1s spectrum. Grey areas depict bonds in the heptazine ring, blue areas the $\mathrm{NH}_{\mathrm{x}}$ or $\mathrm{OH}$ bonds, and orange the $\mathrm{NO}$ or $\mathrm{CO}$ bonds.

XPS analysis of pristine H-PHI and a $2 \mathrm{~h}$ and $24 \mathrm{~h}$ sonicated sample was used to confirm the functional groups introduced into the system by sonication. In C 1s spectra, besides the typical heptazine ring signals two distinct signals are observed at 286.4 and $288.9 \mathrm{eV}$, which can be correlated to $\mathrm{C}-\mathrm{N}\left(\mathrm{H}_{\mathrm{x}}\right)$ or $\mathrm{C}-\mathrm{O}(\mathrm{H})$ (blue area) and $\mathrm{C}=\mathrm{O}$ (orange), respectively, where the latter is assigned either to a carboxylic acid or $\left(\mathrm{NH}_{2}\right)_{\mathrm{x}} \mathrm{C}=\mathrm{O}$ moiety (Figure $2 \mathrm{e}$ ). ${ }^{[6,7,33,34]}$ The formation of vacancies due to sonication can be excluded or considered insignificant (Chapter 5.4, Table S9, Supporting Information). To disentangle the $\mathrm{C}-\mathrm{N}(\mathrm{H})_{\mathrm{x}}$ and $\mathrm{C}-\mathrm{O}(\mathrm{H})$ signals, $\mathrm{N} 1 \mathrm{~s}$ and $\mathrm{O}$ 1s spectra were analyzed as well (Figure 2f, Figure S19, Supporting Information). The relative portion of $\mathrm{NH}_{\mathrm{x}}$ (blue area in the $\mathrm{N} 1 \mathrm{~s}$ XPS spectrum) ${ }^{[6,7,34]}$ increases by up to $13 \%$ with decreasing particle size (Chapter 5.4, Supporting Information), akin to FT-IR and NMR analysis. However, the signal in the $\mathrm{O} 1 \mathrm{~s}$ spectrum at $531.8 \mathrm{eV}(\mathrm{OH} \text { groups, blue area })^{[6,7,35]}$ is reduced with decreasing particle size (to 64\%), probably caused by evaporation of structural water in the vacuum chamber of XPS, which is more likely to happen for smaller particles. 
In addition, a slight increase of $\mathrm{N}-\mathrm{O}$ (orange) (up to $20 \%$, extracted from $\mathrm{N} 1 \mathrm{~s}$ spectrum) and $\mathrm{C}=\mathrm{O}$ bonds (orange) (up to $8 \%$, estimated from $\mathrm{C} 1 \mathrm{~s}$ spectrum) can be found with decreasing particle size. The presence of these groups is also confirmed by O 1s XPS spectra, although here, contributions from the substrate may have a small influence (Chapter 5.4, Supporting Information). The concentration of $\mathrm{N}-\mathrm{O}$ bonds is only around $10 \%$ of the $\mathrm{C}=\mathrm{O}$ concentration (in $\mathrm{COOH}$ or $\left.\mathrm{CO}\left(\mathrm{NH}_{\mathrm{x}}\right)_{\mathrm{y}}\right)$, so that FT-IR spectroscopy shows a slight increase of $\mathrm{C}=\mathrm{O}$ vibrations but no clear trend for N-O (Figure S16-S18, Table S7, Supporting Information). Furthermore, a small peak in ${ }^{13} \mathrm{C}$ NMR at $170 \mathrm{ppm}$ (Figure 2b, S23, Supporting Information) and additional vibrations at 2100 $\mathrm{cm}^{-1}$ in FT-IR (Figure S15-S18, Supporting Information) hint to a meagerly increased amount of $\mathrm{C} \equiv \mathrm{N}$ in sonicated samples. ${ }^{[3,6]}$ In comparison to the other functional groups, the concentration of $\mathrm{N}-\mathrm{C} \equiv \mathrm{N}$ groups in all fractions (pristine-P4) is small and does not significantly change with the particle size.

From these characterizations, we conclude that the ultrasound treatment is sufficiently mild such that the pristine and sonicated PHI are chemically identical in terms of their polymer backbone after sonication. Besides, the particle size reduction with increasing sonication time, the deagglomeration of PHI crystallites in ambient to humid environment increases especially the amount of terminal $\mathrm{OH}$ and $\mathrm{NH}_{2}$ groups (up to $47 \%$ in $\mathrm{P} 4$ compared to the pristine material). Besides $\mathrm{C}=\mathrm{O}$ groups, which rise up to $20 \%$ by particle size reduction from pristine $\mathrm{PHI}$ to $\mathrm{P} 4$, a small amount of $\mathrm{C} \equiv \mathrm{N}$ and $\mathrm{NO}$ defects are formed, which are not severely affected in their concentration by particle size reduction.

\section{Influence of functional groups on photocatalytic hydrogen evolution}

Next, we investigated photocatalytic hydrogen evolution to study the influence of morphological and structural modifications induced by sonication. First, the effects of particle size on the absorption edge of the samples were analyzed. In the pristine material, the optical band gap for $\mathrm{K}-\mathrm{PHI}$ is $2.73( \pm 0.01) \mathrm{eV}$, whereas for H-PHI a value of $2.92( \pm 0.01) \mathrm{eV}$ was obtained. A slight increase in the optical band gap is observed for particle sizes below $200 \mathrm{~nm}$ by $0.09( \pm 0.01) \mathrm{eV}$ (K-PHI) and $0.03( \pm 0.01) \mathrm{eV}(\mathrm{H}-\mathrm{PHI})$, respectively (Figure S25-S28, Table S10, Supporting Information). When reducing the particle size, a pronounced Urbach tail is visible in the UV-Vis spectra. This is likely caused by excitations below the band gap, due to emerging intermediate 
states in the band gap associated with defects, in line with the findings of increasing amounts of surface groups. ${ }^{[36]}$

All PHI materials were tested for the photocatalytic activity in hydrogen evolution reaction (HER) in a $1 \mathrm{mg} \mathrm{mL}^{-1}$ suspension containing $10 \mathrm{vol} \%$ methanol as an electron donor and an optimized $\mathrm{Pt}$ loading of $2 \mathrm{wt} \%$ for H-PHI and $8 \mathrm{wt} \%$ for K-PHI, ${ }^{[3,6,7]}$ photo-deposited from hexachloroplatinic acid during illumination with simulated sunlight (AM 1.5 G). For pristine H-PHI, a maximum activity of $31 \mu \mathrm{mol} \mathrm{h}{ }^{-1}$ was measured, which increases to $35-41 \mu \mathrm{mol} \mathrm{h}^{-1}$ (by 13-25\%) when the average particle sizes are reduced down to 500-180 nm (P1, P2) (Figure S29, Supporting Information). A further reduction in particle size causes a monotonous decrease in HER activity by $97 \%\left(1 \mu \mathrm{mol} \mathrm{h}^{-1}\right)$ for the $24 \mathrm{~h}$ sonicated particles $\mathrm{P} 4$ with an average size of $75 \mathrm{~nm}$ (Figure 3a, S27, Supporting Information). Interestingly, the photocatalytic performance of P4 particles can be increased upon re-agglomeration prior to photo-deposition of $\mathrm{Pt}$, which also works for reagglomerated P1 particles (Figure 3a, S29-S31, Supporting Information), vide infra. Due to drying, P4 particles agglomerate randomly, similar to the coiling process described for carbon nitrides in the literature. ${ }^{[37]}$ The enhanced photocatalytic activity is contrary to the expectation that high dispersity and/or smaller particle sizes lead to a better catalytic rate. ${ }^{[38]}$ For K-PHI, initially, a similar trend is observed (Figure 3b, S30, S33, Supporting Information), although K-PHI is not stable during photocatalysis and transforms into H-PHI, which is assumed to take place faster for smaller particles, causing an increase in photocatalytic activity for small particles again (Figure S32-S34, S45, Supporting Information). For this reason, the following analysis will mainly concentrate on the H-PHI results.

Next, we investigated the influence of particle size on the distribution, size, and loading of the Pt co-catalyst, which typically is a "blind spot" in most reported photocatalytic experiments with carbon nitrides. As a general trend, we observe a decrease in the size of Pt nanoparticles, when the PHI particle size is reduced (Figure S37-S43, Supporting Information). At the same time, the Pt particles are more homogeneously distributed (Figure 3c-e). For pristine H-PHI, the Pt particle size was estimated by SEM to be in the range of 8-38 nm (Figure 3c, Figure S37, S40, Supporting Information) while it increases in P1 up to $40-68 \mathrm{~nm}$. When decreasing the particle size further to P2-P4, the Pt particle size is reduced to 1-5 nm (Figure 3d, e, S37-S40, Supporting Information). The different Pt particle sizes likely correlate with the nature and number of PHI functional groups: 
with decreasing particle size, the number of surface functional groups increases, which can act as Pt coordination-centers and nucleation sites (Figure S40-S43 Supporting Information). In addition, the more Pt nucleation sites are present, the more homogeneous the Pt coverage, which has a positive effect on the photocatalytic activity, as has also been reported for melon-based carbon nitrides, since charge carrier migration distances are very limited. ${ }^{[2,39]}$ In line with this, a shift by 0.40-0.45 eV to lower binding energies can be observed for the Pt 4f XPS signals correlated with $\mathrm{Pt}^{2+}$ for $\mathrm{P} 4$ in comparison to pristine $\mathrm{H}-\mathrm{PHI}$, which is likely caused by stronger metal-support interactions via surface $\mathrm{NH}, \mathrm{OH}$, and $\mathrm{CO}$ groups (Figure S19, Chapter 5.4, Supporting Information). ${ }^{[6,15,40]}$ The stronger the metal-carbon nitride interaction, the better the local charge transfer. ${ }^{[15,41]}$ In addition, the XPS spectra showed a decreasing $\mathrm{Pt}^{2+} / \mathrm{Pt}^{0}$ ratio up to $20 \%$ for decreasing particle sizes (pristine material: 1.70; P4: 1.37) (Table S9, Supporting Information), which points to an incomplete Pt reduction for bigger particles, possibly due to less efficient overall charge transfer. ${ }^{[42]}$
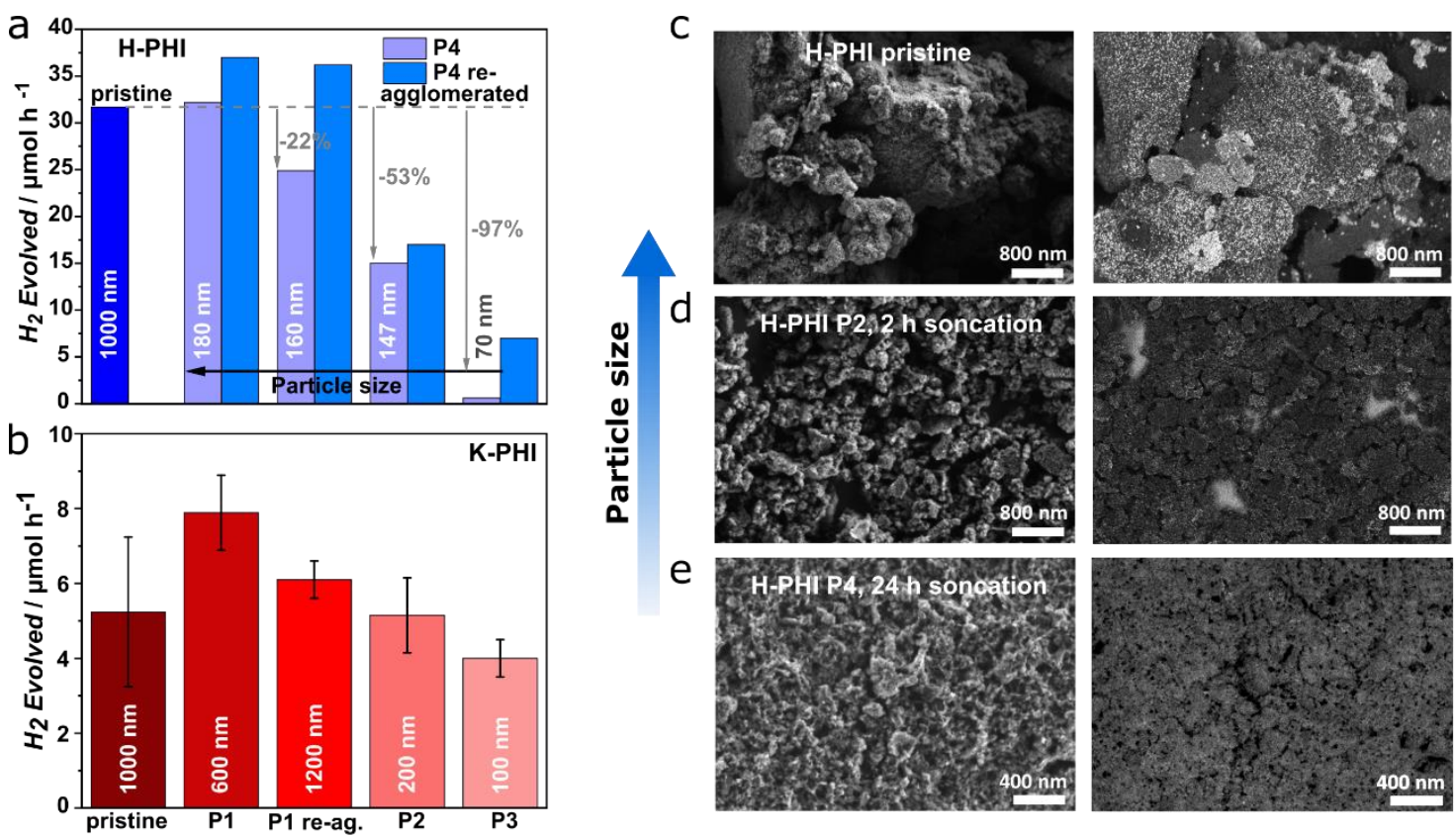

Figure 3: Photocatalytic hydrogen evolution of H-PHI and K-PHI in the presence of the electron donor methanol (10 vol\%) and co-catalyst Pt (2 wt\% for H-PHI and $8 \mathrm{wt} \%$ for K-PHI). a) Hydrogen evolution in the presence of H$\mathrm{PHI}$ pristine and $\mathrm{P} 4$ (24 h sonication) as well as re-agglomerated $\mathrm{P} 4$ as a function of average particle size in batch (20 $\mathrm{mg}$ of catalyst were used). b) Hydrogen evolution of K-PHI pristine, P1, P1 re-agglomerated, P2 and P3 (5 mg of catalyst were used). SEM images of H-PHI after photocatalysis (left) and back-scattered electron image with brighter spots displaying Pt (right) c) for pristine H-PHI, d) for 2 h sonicated (P2) H-PHI and e) for $24 \mathrm{~h}$ sonicated (P4) HPHI.

Regarding the Pt loading, we found that with decreasing particle size (down to a particle size of $\sim 250 \mathrm{~nm}$ ), more Pt was photo-deposited on the material. While $2 \mathrm{wt} \%$ added Pt were deposited on 
particles of $250 \mathrm{~nm}$ in size, only $1 \mathrm{wt} \% \mathrm{Pt}$ was found on pristine particles after photocatalysis (Table S11, Supporting Information). At smaller sizes below $250 \mathrm{~nm}$ the Pt amount decreases again (1.4 wt\% at $79 \mathrm{~nm}$ particle size) (Table S11, Figure S41-S44, Supporting Information). The fact that less Pt is deposited on smaller particles despite the larger amount of functional groups and enhanced local metal-carbon nitride interaction points to a faster recombination process of photo-generated charges in smaller particles. Such enhanced recombination rates are detrimental for HER (Figure S36, S44 Supporting Information) as observed for particle sizes < $180 \mathrm{~nm}$, which are probably influenced by the amount and type of surface functional groups (especially $\mathrm{NH}_{2}, \mathrm{OH}$ and $\mathrm{C}=\mathrm{O}$ ) (Figure S35, Supporting Information). In the pristine material, however, the bulk-based charge separation across the $2 \mathrm{D}$ layers ${ }^{[12,17]}$ appears to be hindered by the long diffusion lengths of the photo-generated charges to reach the catalytic surface, leading to decreased activity. The charge diffusion might be enhanced in re-agglomerated particles due to the nature and density of functional groups at their surface or interface, ${ }^{[12,43]}$ which is resulting in an enhanced photocatalytic activity.
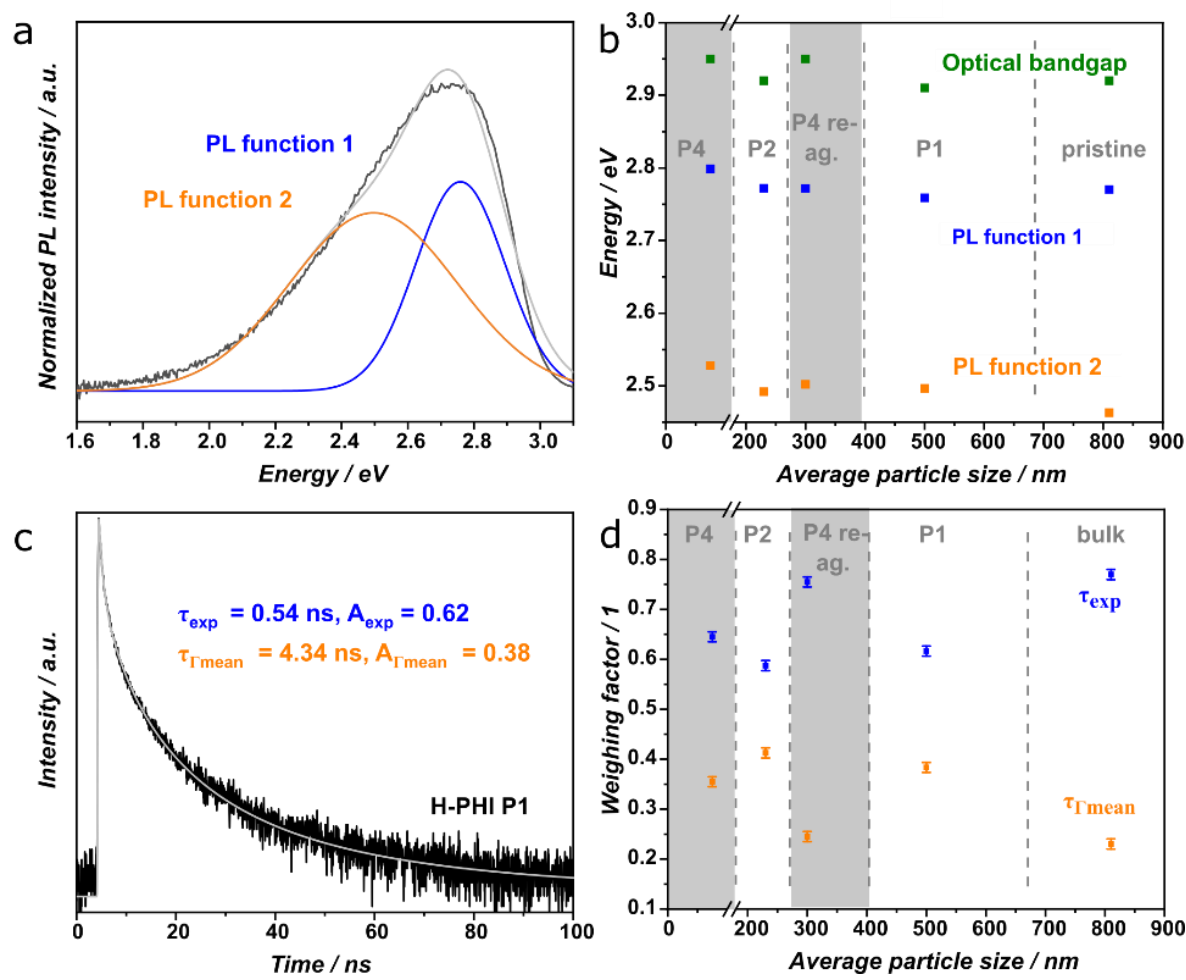

Figure 4: Photoluminescence (PL) analysis of H-PHI in degassed water. a) Deconvolution of the PL signal of H-PHI P1 based on two Gaussian functions to describe also shallow and deep states in the band gap. b) The optical band gap of H-PHI and PL signal deconvolution by two Gaussian functions depending on the particle size. Re-agglomerated particles are denoted as "re-ag.". c) Time-dependent PL analysis of H-PHI P1, which is fitted with one exponential and a $\Gamma$ function. $d$ ) Contribution of the exponential and $\Gamma$-decay to the overall lifetime decay (errors included) as a function of the average batch particle size. 
To study the influence of the surface functional groups and their possible role as trap states, which have already been suggested to be present by UV-vis spectroscopy, we used photoluminescence (PL) spectroscopy. ${ }^{[23]} \mathrm{PL}$ experiments were performed on the pristine material in degassed water, degassed water and electron donor and under the same conditions as photocatalysis experiments, i.e. in the presence of an electron donor and co-catalyst (Figure S46, Supporting Information). Figure 4a shows a representative emission spectrum of H-PHI P1 in degassed, $\mathrm{O}_{2}$-free water, excited at $370 \mathrm{~nm}$, where the maximum is located at around $450 \mathrm{~nm}(2.7 \mathrm{eV})$. The broad PL signal can be deconvoluted into two Gaussian contributions, similar to carbon nitride materials reported in the literature, ${ }^{[23,36]}$ located at around $2.75 \mathrm{eV}$, stemming from the bulk, and at $2.45 \mathrm{eV}$, corresponding to mostly (surface) trap states (Figure 4a, S46-S49, Supporting Information). ${ }^{[23]}$ Both emission peaks are smaller than the optical band gap of $2.9 \mathrm{eV}$ for all studied environments, indicating that emissions are caused by states in the band gap. Although their spectral positions slightly vary with the particle size investigated, their energetic shifts are constant relative to the band gap, as shown in Figure 4b.

In order to extract more information about the influence of the trap states, time-resolved photoluminescence (TRPL) measurements were carried out, which contain information on both radiative and non-radiative decay types. ${ }^{[44]}$ These were fitted by a combination of an exponential and a $\Gamma$-function (Figure 4c, S50, Supporting Information). ${ }^{[10]}$ A priori, both recombination types can show a distribution decay nature according to a $\Gamma$ function ${ }^{[45]}$ However, the best fit obtained in all cases is an exponential function attributed to the faster decay in the bulk ( $<0.15 \mathrm{~ns})$. The distribution function $(\Gamma)$, which accounts for all decays on longer time scale, is assigned to trap states induced by surface terminations. ${ }^{[46,47]}$ The respective weight factors of both processes are compared for their contribution to the excited charge carrier decay process as a function of particle size (Figure 4d, S50, Table S12, Supporting Information). In aqueous suspension, where the intrinsic properties are best visible (Figure 4b, d), an increase in the relative weight attributed to the traps (orange) is observed as PHI is sonicated. This finding is consistent with an increase of functional groups on the particle's surface that can act as trap states. Additionally, shorter lifetimes were observed with smaller particle sizes (Table S12, Supporting Information), probably due to enhanced formation and population of such trap states, yielding a higher degree of charge recombination or capturing the charges on longer timescales exceeding the time scale of the PL 
measurement. Under the conditions in which the photocatalysis experiments take place, i.e. donor presence and photo-deposited Pt, the relative PL trends for the surface based processes are preserved (Figure S51, S52, Supporting Information). However, the absolute numbers are changing. Charge trapping and recombination in the bulk is not affected by the addition of Pt.

The re-agglomeration of $\mathrm{P} 4$ particles prolongs the lifetime compared to that of the pristine material, significantly (Table S12, Supporting Information). The relative weight of the PL fit functions reaches similar values to that of the pristine material (Figure 4d). This can be rationalized by an enhanced exciton separation in the agglomerates. ${ }^{[12]}$ The transfer between crystalline areas might even be enhanced in the re-agglomerated samples due to more or different functional groups at the interface and hence possible hydrogen bonding between the agglomerated particles. ${ }^{[28,48]}$ As evidenced by electrochemical impedance spectroscopy, a grain-boundary based electron transport process is likely to be present, which hints to a multiple-trapping-and-release model (MTR)-like process in PHI (Figure S53, Supporting Information). The electrons are transported across the layers in the crystallites, and once reaching an edge of the crystallite, charge hopping becomes necessary. The hopping is enhanced by shallow trap states formed at the interface and improves with well-interconnected interfaces. ${ }^{[28]}$ Similar results were reported for a melon-type carbon nitride, where enhanced exciton dissociation was reported at the interface between ordered and disordered carbon nitride structure. ${ }^{[49]}$

To better estimate the energetics of the functional groups $\left(\mathrm{NH}_{2}, \mathrm{OH}, \mathrm{NHC} \equiv \mathrm{N}\right.$ and $\mathrm{NHCOOH}$ or $\mathrm{NHCONH}_{2}$ ) and hence, trap states for different particle sizes, quantum-chemical calculations were performed, which provide insights into the nature of the trap states and their energetic depth. Three different model systems for PHI were designed as size-increasing computational models, consisting of 1, 7, and 19 pores to approach the different particle sizes that are actually observed under experimental conditions (Figure S54 Supporting Information). When comparing the localization of HOMO and LUMO, no significant difference was found for the localization as a function of the size of the model system or functional group (Figure S55-S61, Table S13 Supporting Information). Independent of the size of the pore model, orbitals are rather localized at single hepatize units than being delocalized over the entire system (Figure S55-S57, Table S13, Supporting Information), hence revealing contributions from functional groups at the pore edges. Changes in orbital contributions by functional groups are also reflected in the calculated optical 
band gaps, where the largest values were found for primary amine terminations with $4.35 \mathrm{eV}$, followed by $\mathrm{NHCONH}_{2}(4.31 \mathrm{eV})$, hydroxyl $(4.30 \mathrm{eV}), \mathrm{NHC} \equiv \mathrm{N}(4.27 \mathrm{eV})$ and $\mathrm{NHCOOH}$ groups (4.25 eV), successively decreasing the band gap (Table S14, Supporting Information). The smaller the experimentally analyzed PHI particles are, the more significant the influence of the functional groups. However, it is likelier that the functional groups form trap states, rather than a homogenous shifts of the band edges. This is reflected by both the Urbach tail seen in the UV-Vis spectra (Figure S26, Supporting Information) and by spectral PL deconvolution (Figure S47, Supporting Information). In addition, calculated ionization potentials $\left(\mathrm{E}_{\mathrm{IP}}\right)$ and electron affinities $\left(\mathrm{E}_{\mathrm{EA}}\right)$ for different model systems (Chapter 6.6 Supporting Information) were identified as proxies for the functional group contribution and band edge energies (Figure S62-S66, Supporting Information).

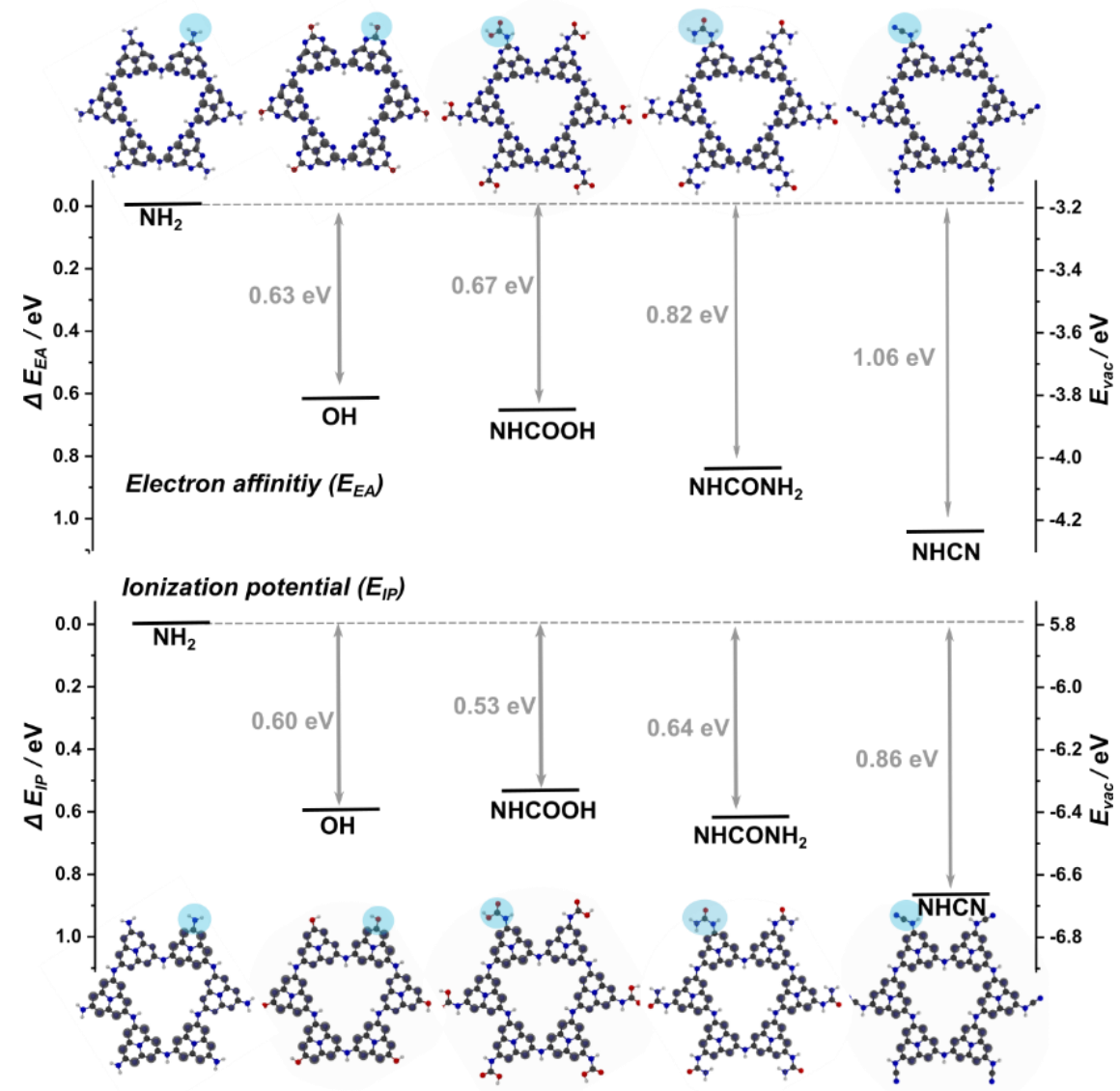

Figure 5: Quantum-chemical calculations of electron affinity $\left(\mathrm{E}_{\mathrm{EA}}\right)$ and ionization potential $\left(\mathrm{E}_{\mathrm{IP}}\right)$ of the one-pore PHI model system obtained on PBE0-D3/def2-TZVP ${ }^{[50]} / / \mathrm{RI}-\mathrm{PBE}-\mathrm{D} 3 /$ def2-TZVP ${ }^{[51]}$ level of theory. Comparison of different terminal groups ( $\mathrm{NH}_{2}$, $\mathrm{OH}, \mathrm{NHCOOH}, \mathrm{NHCONH}_{2}$ and $\mathrm{NHC} \equiv \mathrm{N}$ ), with values referenced to the $\mathrm{NH}_{2}$ terminal group.

Akin to the literature, it was found that relative to terminal $\mathrm{NH}_{2}$ groups, the $\mathrm{OH}$ groups change the $\mathrm{E}_{\mathrm{IP}}\left(\right.$ by $0.60 \mathrm{eV}$ ) and $\mathrm{E}_{\mathrm{EA}}$ (by $0.63 \mathrm{eV}$ ) in a similar way such that only shallow trap states are formed 
close to the LUMO (Figure 5, S67, Supporting Information). ${ }^{[23,52]} \mathrm{C}=\mathrm{O}$ and $\mathrm{C} \equiv \mathrm{N}$ containing terminal groups reduce the optical band gap the most, which is also visible in a more pronounced shift of the $\mathrm{E}_{\mathrm{EA}}$ in comparison to $\mathrm{E}_{\mathrm{IP}}$ relative to the $\mathrm{NH}_{2}$ terminated system (Figure S68-S70, Supporting Information). This might be consistent with the formation of trap states that are located deeper in the optical band gap than for $\mathrm{NH}_{2}$ and $\mathrm{OH}$ groups. ${ }^{[22-24]}$ For this reason $\mathrm{NHCOOH}$, $\mathrm{NHCONH}_{2}$ and $\mathrm{NHC} \equiv \mathrm{N}$ groups might influence the charge carrier energetics, and therefore the photocatalytic activity, most profoundly.

The higher the concentration of functional groups in general, the higher the amount of detrimental deeper trap states that would enhance recombination and hence reduce the hydrogen evolution rate. ${ }^{[24,47]}$ In agreement with photocatalysis experiments and PL data, the highest photocatalytic activity was measured for P1 and P2 samples, with a slight higher but not excessive amount of functional groups as evidenced by structural analysis. Based on these findings, we conclude that it is possible to tune the light absorption properties and the amount and depth of trap states by introducing different functional groups intentionally, which has a pronounced influence on the availability of charge carriers for HER.

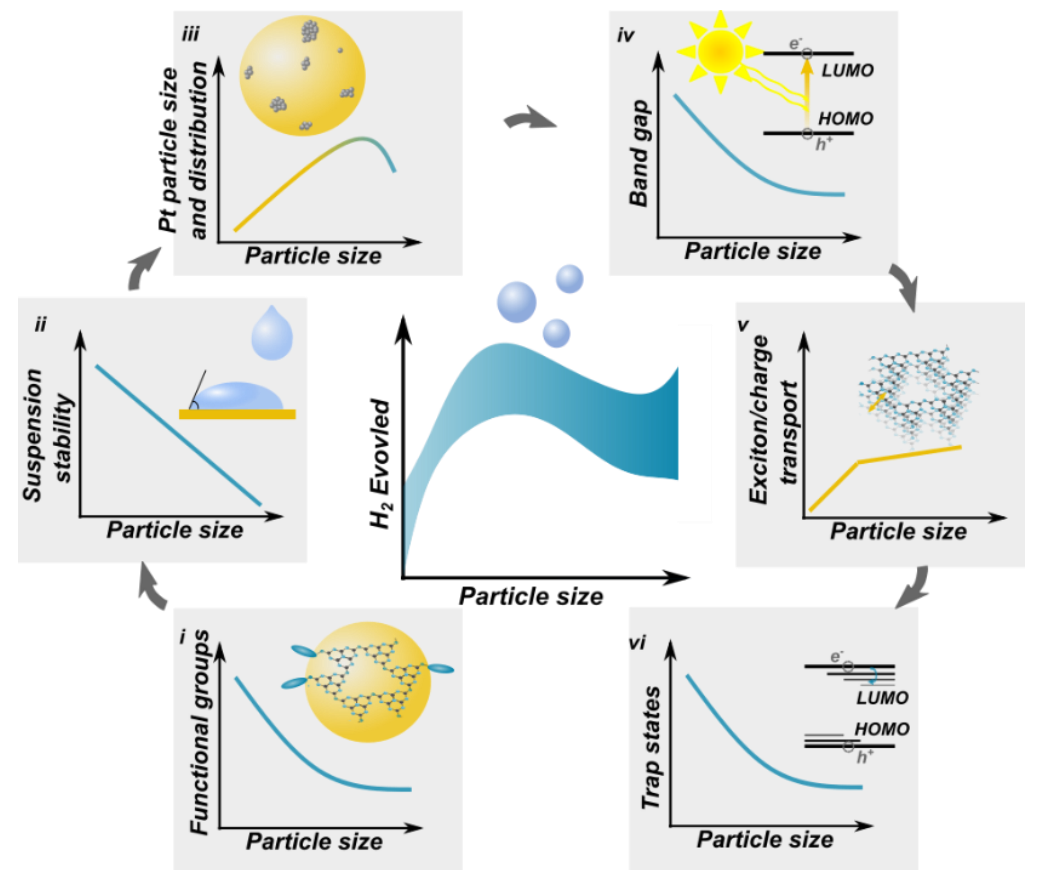

Figure 6: Overview of different contributions to hydrogen evolution, including the concentration of functional groups (i), suspension stability (ii), Pt particle size and distribution (iii), light absorption (iv), exciton separation and transport (v), and amount and depth of trap states (vi). With different effects on hydrogen evolution, a combination of those contributions is responsible for the observed hydrogen evolution trend. 
Taking together the results for sonicated PHI and their effects on photocatalysis, it can be stated that photocatalysis is a process with multiple interwoven parameters. ${ }^{[27]}$ They act together on the hydrogen evolution activity in different and partly opposing ways. By changing the size of the photocatalyst, e.g. by sonication, the amount of functional groups, the particle dispersibility, the size, distribution and loading of the Pt co-catalyst, light absorption, exciton or charge separation, as well as the amount and depth of trap states are all changed, as summarized in Figure 6. Contrary to most published results, a non-linear trend for the actual measured photocatalytic activity as a particle size function was obtained. This is caused by an increased amount of functional groups (especially $\mathrm{OH}, \mathrm{NH}_{2}$, and $\mathrm{C}=\mathrm{O}$ ) at the surface with decreasing particle sizes (i), which leads to enhanced polarity/wettability and suspension stability (ii). As a result, the Pt deposition is more homogeneous, and the Pt particle size is reduced for smaller PHI particles (iii). However, the band gap increases for very small particles (iv), and the charge or exciton separation is decreased with too small particles due to restrictions in layer number and, therefore, carrier diffusion length (v). Higher levels of deep trap states are observed in smallest size regime due to a larger number of surface defects (vi). These deep lying surface defects reduce the hydrogen evolution activity, as long as bulk recombination is not the limiting factor for large particles. Although a complex interplay of these factors determines the effective photocatalytic activity, the increase in HER rate for particle sizes in the range of $180-500 \mathrm{~nm}$ suggests that tuning individual parameters is indeed possible. This way, beneficial properties become exposed while limiting effects become less detrimental. This approach provides a general template for future catalyst design.

\section{Conclusion}

In this work, we have used ultrasonication as a route to systematically study correlations between morphology-related properties and photocatalytic hydrogen evolution rates in the $2 \mathrm{D}$ carbon nitride poly(heptazine imide). By reducing the particle size of PHI down to $20 \mathrm{~nm}$ by deagglomeration, this technique successfully yields stable colloidal solutions while preserving the structural integrity of the PHI primary crystallites in the in-plane as well as the out-of-plane directions. A combined structural analysis by NMR, FT-IR, PXRD, TEM, and XPS revealed that the polymer backbone remains unchanged when reducing particle size, and that the number of functional groups (mainly $\mathrm{NH}_{2}, \mathrm{OH}$, and $\mathrm{C}=\mathrm{O}$ bearing) located at the periphery increases. As a result, the Pt distribution becomes more homogeneous. This highlights the intertwined effects resulting from size reduction and their impact on charge carrier localization, co-catalyst 
attachment, and consequently, charge transfer for HER. TRPL measurements in combination with electrochemical analysis and quantum-chemical calculations suggest that the increasing amount of functional groups creates trap states, which are only beneficial for photocatalytic hydrogen evolution as long as their influence is not excessive. Thus, an optimum activity is reached for $2 \mathrm{~h}$ sonicated samples with a particle size of 180-500 $\mathrm{nm}$ and re-agglomerated samples of those particles. The functional groups formed are also beneficial for charge transport at particle interfaces, consistent with a multiple-trapping-and-release (MTR) model known for semicrystalline organic semiconductors. If particles are too small, an excess amount of recombination centers are introduced, likely caused by defects lying deep in the band gap. These trap states reduce the efficiency for Pt deposition and hence charge transfer, resulting in lower photocatalytic activity as evidenced by quantum-chemical calculations of ionization potentials and electron affinities. For particles below $180 \mathrm{~nm}$, large optical band gaps and deep trap states adversely affect photocatalysis, whereas the remaining effects of size reduction such as increased wettability enhance photocatalytic efficiency. The results presented here are not only instrumental in the context of PHI thin film and device fabrication, but they might also help to tune the properties of photocatalytic carbon nitride based microswimmers or solar battery applications. Disentangling the interdependent structural, morphological, and optoelectronic variables, which together define light-induced charge transport and transfer reactions is key to understand, address and optimize general photocatalyst design.

\section{Acknowledgements}

Financial support by the Max Planck Society, the ERC Starting Grant (project COFLeaf, grant number 639233), the Center for NanoScience, DFG cluster of excellence "e-conversion” (EXC 2089/1-390776260) is gratefully acknowledged. C.O. acknowledges financial support as a Max Planck Fellow at the Max Planck Institute for Solid State Research, Stuttgart. A.J.S. gratefully acknowledges a postdoctoral scholarship from the Max Planck Society. The authors thank S. Emmerling for BET measurements.

\section{References}

[1] a) X. Wang, K. Maeda, A. Thomas, K. Takanabe, G. Xin, J. M. Carlsson, K. Domen, M. Antonietti, Nat. Mater. 2009, 8 (1), 76-80; b) J. Liu, H. Wang, M. Antonietti, Chem. Soc. Rev. 2016, 45 (8), 2308-2326.

[2] T. Banerjee, F. Podjaski, J. Kröger, B. P. Biswal, B. V. Lotsch, Nat. Rev. Mater. 2021, 6 (2), 168190. 
[3] H. Schlomberg, J. Kröger, G. Savasci, M. W. Terban, S. Bette, I. Moudrakovski, V. Duppel, F. Podjaski, R. Siegel, J. Senker, R. E. Dinnebier, C. Ochsenfeld, B. V. Lotsch, Chem. Mater. 2019, 31 (18), 7478-7486.

[4] K. Schwinghammer, M. B. Mesch, V. Duppel, C. Ziegler, J. Senker, B. V. Lotsch, J. Am. Chem. Soc. 2014, 136 (5), 1730-1733.

[5] Z. Chen, A. Savateev, S. Pronkin, V. Papaefthimiou, C. Wolff, M. G. Willinger, E. Willinger, D. Neher, M. Antonietti, D. Dontsova, Adv. Mater. 2017, 29 (32), 1700555.

[6] V. W.-h. Lau, I. Moudrakovski, T. Botari, S. Weinberger, M. B. Mesch, V. Duppel, J. Senker, V. Blum, B. V. Lotsch, Nat. Commun. 2016, 7 (1), 12165.

[7] V. W.-h. Lau, V. W.-z. Yu, F. Ehrat, T. Botari, I. Moudrakovski, T. Simon, V. Duppel, E. Medina, J. K. Stolarczyk, J. Feldmann, V. Blum, B. V. Lotsch, Adv. Energy Mater. 2017, 7 (12), 1602251.

[8] a) V. W.-h. Lau, D. Klose, H. Kasap, F. Podjaski, M.-C. Pigne, E. Reisner, G. Jeschke, B. V. Lotsch, Angew. Chem. Int. Ed. 2017, 56, 510-514; b) H. Kasap, C. A. Caputo, B. C. M.

Martindale, R. Godin, V. W.-H. Lau, B. V. Lotsch, J. R. Durrant, E. Reisner, J. Am. Chem. Soc.

2016, 138, 9183-9192.

[9] F. Podjaski, J. Kröger, B. V. Lotsch, Adv. Mater. 2018, 30 (9), 1705477.

[10] J. Kröger, A. Jiménez-Solano, G. Savasci, P. Rovó, I. Moudrakovski, K. Küster, H. Schlomberg, H. A. Vignolo-González, V. Duppel, L. Grunenberg, C. B. Dayan, M. Sitti, F. Podjaski, C. Ochsenfeld, B. V. Lotsch, Adv. Energy Mater. 2021, 11 (6), 2003016.

[11] a) L. Liang, L. Shi, F. Wang, Diamond Relat. Mater. 2019, 91, 230-236; b) L. Svoboda, P. Praus, M. J. Lima, M. J. Sampaio, D. Matýsek, M. Ritz, R. Dvorský, J. L. Faria, C. G. Silva, Mater. Res. Bull. 2018, 100, 322-332.

[12] K. L. Corp, C. W. Schlenker, J. Am. Chem. Soc. 2017, 139 (23), 7904-7912.

[13] S. Yang, Y. Gong, J. Zhang, L. Zhan, L. Ma, Z. Fang, R. Vajtai, X. Wang, P. M. Ajayan, Adv. Mater. 2013, 25 (17), 2452-2456.

[14] X. Dong, F. Cheng, J. Mater. Chem. A 2015, 3 (47), 23642-23652.

[15] Y. Shiraishi, Y. Kofuji, S. Kanazawa, H. Sakamoto, S. Ichikawa, S. Tanaka, T. Hirai, Chem. Commun. 2014, 50 (96), 15255-15258.

[16] Q. Han, B. Wang, J. Gao, Z. Cheng, Y. Zhao, Z. Zhang, L. Qu, ACS Nano 2016, 10 (2), 27452751.

[17] C. Merschjann, S. Tschierlei, T. Tyborski, K. Kailasam, S. Orthmann, D. Hollmann, T. SchedelNiedrig, A. Thomas, S. Lochbrunner, Adv. Mater. 2015, 27 (48), 7993-7999.

[18] P. Merkl, F. Mooshammer, P. Steinleitner, A. Girnghuber, K. Q. Lin, P. Nagler, J. Holler, C. Schüller, J. M. Lupton, T. Korn, S. Ovesen, S. Brem, E. Malic, R. Huber, Nat. Mater. 2019, 18 (7), 691-696.

[19] a) Z. Xue, F. Liu, J. Jiang, J. Wang, T. Mu, Green Chem. 2017, 19 (21), 5041-5045; b) M. J. Bojdys, N. Severin, J. P. Rabe, A. I. Cooper, A. Thomas, M. Antonietti, Macromol. Rapid Commun. 2013, 34 (10), 850-854; c) Z. Zhou, J. Wang, J. Yu, Y. Shen, Y. Li, A. Liu, S. Liu, Y. Zhang, J. Am. Chem. Soc. 2015, 137 (6), 2179-2182; d) F. Cheng, H. Wang, X. Dong, Chem. Commun. 2015, 51 (33), 7176-7179; e) M. Wu, J.-M. Yan, X.-n. Tang, M. Zhao, Q. Jiang, ChemSusChem 2014, 7 (9), 2654-2658.

[20] a) W. Ren, J. Cheng, H. Ou, C. Huang, M.-M. Titirici, X. Wang, ChemSusChem 2019, 12 (14), 3257-3262; b) P. Niu, M. Qiao, Y. Li, L. Huang, T. Zhai, Nano Energy 2018, 44, 73-81; c) J. Zhang, J. Chen, Y. Wan, H. Liu, W. Chen, G. Wang, R. Wang, ACS Appl. Mater. Inter. 2020, 12 (12), 13805-13812.

[21] H. Yu, R. Shi, Y. Zhao, T. Bian, Y. Zhao, C. Zhou, G. I. N. Waterhouse, L.-Z. Wu, C.-H. Tung, T. Zhang, Adv. Mater. 2017, 29 (16), 1605148.

[22] Y. Wang, P. Du, H. Pan, L. Fu, Y. Zhang, J. Chen, Y. Du, N. Tang, G. Liu, Adv. Mater. 2019, 31 (40), 1807540. 
[23] R. Godin, Y. Wang, M. A. Zwijnenburg, J. Tang, J. R. Durrant, J. Am. Chem. Soc. 2017, 139 (14), 5216-5224.

[24] J. Xue, M. Fujitsuka, T. Majima, Phys. Chem. Chem. Phys. 2019, 21 (5), 2318-2324.

[25] a) P. Wu, J. Wang, J. Zhao, L. Guo, F. E. Osterloh, J. Mater. Chem. A 2014, 2 (47), 2033820344; b) H. Ou, L. Lin, Y. Zheng, P. Yang, Y. Fang, X. Wang, Adv. Mater. 2017, 29 (22), 1700008.

[26] J. Huang, Y. Cao, H. Wang, H. Yu, F. Peng, H. Zou, Z. Liu, Chem. Eng. J. 2019, 373, 687-699.

[27] K. Takanabe, ACS Catal. 2017, 7 (11), 8006-8022.

[28] O. Simonetti, L. Giraudet, Polym. Int. 2019, 68 (4), 620-636.

[29] M. Lotya, A. Rakovich, J. F. Donegan, J. N. Coleman, Nanotechnology 2013, 24, 265703.

[30] K. S. W. Sing, Pure Appl. Chem. 1985, 57 (4), 603.

[31] N. Pokhrel, P. K. Vabbina, N. Pala, Ultrason. Sonochem. 2016, 29, 104-128.

[32] S. J. Makowski, P. Köstler, W. Schnick, Chem.- Eur. J. 2012, 18 (11), 3248-3257.

[33] a) J. Oh, R. J. Yoo, S. Y. Kim, Y. J. Lee, D. W. Kim, S. Park, Chem.-Eur. J. 2015, 21 (16), 6241-6246; b) F. T. Johra, J.-W. Lee, W.-G. Jung, J. Ind. Eng. Chem. 2014, 20 (5), 2883-2887; T. P. Fehlner, J. Organomet. Chem. 1977, 132 (1), C9-C10; c) T. S. Miller, A. B. Jorge, T. M. Suter, A. Sella, F. Corà, P. F. McMillan, Phys. Chem. Chem. Phys. 2017, 19 (24), 15613-15638.

[34] a) H. Katsumata, F. Higashi, Y. Kobayashi, I. Tateishi, M. Furukawa, S. Kaneco, Sci. Rep. 2019, 9 (1), 14873; b) J. Chen, X. Xu, T. Li, K. Pandiselvi, J. Wang, Sci. Rep. 2016, 6 (1), 37318; c) Z. Zhou, Y. Shen, Y. Li, A. Liu, S. Liu, Y. Zhang, ACS Nano 2015, 9 (12), 12480-12487.

[35] C.-C. Hu, M.-S. Wang, W.-Z. Hung, Chem. Eng. Sci. 2017, 167, 1-9.

[36] B. Choudhury, K. K. Paul, D. Sanyal, A. Hazarika, P. K. Giri, J. Phys. Chem. C 2018, 122 (16), 9209-9219.

[37] X. Wei, X. Liu, H. Liu, S. Yang, H. Zeng, F. Meng, X. Lei, J. Liu, Sol. Energy 2019, 181, 161168.

[38] a) X. Wang, L. Chen, S. Y. Chong, M. A. Little, Y. Wu, W.-H. Zhu, R. Clowes, Y. Yan, M. A. Zwijnenburg, R. S. Sprick, A. I. Cooper, Nat. Chem. 2018, 10 (12), 1180-1189; b) Y. Wang, A. Vogel, M. Sachs, R. S. Sprick, L. Wilbraham, S. J. A. Moniz, R. Godin, M. A. Zwijnenburg, J. R. Durrant, A. I. Cooper, J. Tang, Nat. Energy 2019, 4 (9), 746-760.

[39] a) Z. Chen, S. Mitchell, E. Vorobyeva, R. K. Leary, R. Hauert, T. Furnival, Q. M. Ramasse, J. M. Thomas, P. A. Midgley, D. Dontsova, M. Antonietti, S. Pogodin, N. López, J. Pérez-Ramírez, Adv. Funct. Mater. 2017, 27 (8), 1605785; b) N.-N. Vu, S. Kaliaguine, T.-O. Do, ACS Sustain. Chem. Eng. 2020, 8 (2), 853-863; X. Li, W. Bi, L. Zhang, S. Tao, W. Chu, Q. Zhang, Y. Luo, C. $\mathrm{Wu}, \mathrm{Y} . \mathrm{Xie}, A d v$. Mater. 2016, 28 (12), 2427-2431.

[40] W. Xing, W. Tu, M. Ou, S. Wu, S. Yin, H. Wang, G. Chen, R. Xu, ChemSusChem 2019, 12 (9), 2029-2034.

[41] V. W.-h. Lau, D. Klose, H. Kasap, F. Podjaski, M.-C. Pignié, E. Reisner, G. Jeschke, B. V. Lotsch, Angew. Chem. Int. Ed. 2017, 56 (2), 510-514.

[42] S. Cao, J. Jiang, B. Zhu, J. Yu, Phys. Chem. Chem. Phys. 2016, 18 (28), 19457-19463.

[43] a) G. Zhang, G. Li, T. Heil, S. Zafeiratos, F. Lai, A. Savateev, M. Antonietti, X. Wang, Angew. Chem. Int. Ed. 2019, 58 (11), 3433-3437; b) A. Isacsson, A. W. Cummings, L. Colombo, L. Colombo, J. M. Kinaret, S. Roche, 2 D Mater. 2016, 4 (1), 012002; c) Y. Wang, P. Han, X. Lv, L. Zhang, G. Zheng, Joule 2018, 2 (12), 2551-2582; d) H. F. Haneef, A. M. Zeidell, O. D. Jurchescu, J. Mater. Chem. C 2020, 8 (3), 759-787; e) Y. Zhang, Y. Dai, H. Li, L. Yin, M. R. Hoffmann, Commun. Mater. 2020, 1 (1), 66.

[44] A. F. van Driel, I. S. Nikolaev, P. Vergeer, P. Lodahl, D. Vanmaekelbergh, W. L. Vos, Phys. Rev. B 2007, 75 (3), 035329.

[45] A. C. Fogarty, A. C. Jones, P. J. Camp, Phys. Chem. Chem. Phys. 2011, 13 (9), 3819-3830.

[46] R. Brüninghoff, K. Wenderich, J. P. Korterik, B. T. Mei, G. Mul, A. Huijser, J. Phys. Chem. C 2019, 123 (43), 26653-26661. 
[47] W. Yang, R. Godin, H. Kasap, B. Moss, Y. Dong, S. A. J. Hillman, L. Steier, E. Reisner, J. R. Durrant, J. Am. Chem. Soc. 2019, 141 (28), 11219-11229.

[48] a) J. E. Ellis, D. C. Sorescu, S. C. Burkert, D. L. White, A. Star, ACS Appl. Mater. Interfaces 2017, 9 (32), 27142-27151; b) R. Meng, Y. Li, C. Li, K. Gao, S. Yin, L. Wang, Phys. Chem. Chem. Phys. 2017, 19 (36), 24971-24978.

[49] H. Wang, X. Sun, D. Li, X. Zhang, S. Chen, W. Shao, Y. Tian, Y. Xie, J. Am. Chem. Soc. 2017, 139 (6), 2468-2473.

[50] a) C. Adamo, V. Barone, J. Chem. Phys. 1999, 110 (13), 6158-6170; b) M. Ernzerhof, G. E. Scuseria, J. Chem. Phys. 1999, 110 (11), 5029-5036.

[51] a) J. P. Perdew, K. Burke, M. Ernzerhof, Phys. Rev. Lett. 1996, 77 (18), 3865-3868; b) S. Grimme, J. Antony, S. Ehrlich, H. Krieg, J. Chem. Phys. 2010, 132 (15), 154104; c) A. Schäfer, C. Huber, R. Ahlrichs, J. Chem. Phys. 1994, 100 (8), 5829-5835; d) K. Eichkorn, F. Weigend, O. Treutler, R. Ahlrichs, Theor. Chem. Acc. 1997, 97 (1), 119-124.

[52] J. Xue, M. Fujitsuka, T. Majima, Chem. Commun. 2020, 56 (44), 5921-5924.

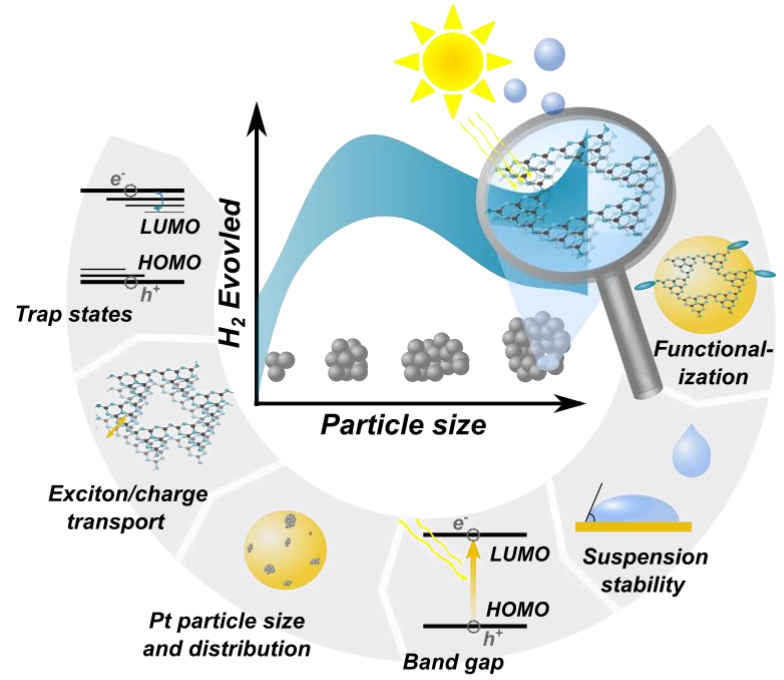

Tuning the particle size of the 2D carbon nitride poly(heptazine imide) enables optimization of photocatalytic hydrogen evolution. We show that changes in the particle size affect the overall photocatalytic process in different ways, and we trace back the individual contributions of sizerelated variables on the photocatalytic activity. This multi-parameter analysis offers design strategies for next-generation photocatalysts. 